\title{
Research Paper: Evaluation of the Effectiveness of Dynamic Neuromuscular Stability Exercises on Balance and Walking Function in the Elderly
}

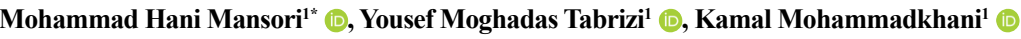 \\ 1. Department of Health and Sport Medicine, Faculty of Physical Education and Sport Sciences, University of Tehran, Tehran, Iran
}

\begin{tabular}{|l|l}
\hline $\begin{array}{c}\text { Use yourdevice to scan } \\
\text { and read the article online }\end{array}$ & $\begin{array}{l}\text { Cftation: Mansori MH, Moghadas Tabrizi Y, Mohammadkhani K. Evaluation of the Effectiveness of Dynamic Neuromus- } \\
\text { cular Stability Exercises on Balance and Walking Function in the Elderly. Iranian Rehabilitation Journal. 2021; 19(3):279-288. } \\
\text { http://dx.doi.org/10.32598/irj.19.3.1406.3 }\end{array}$ \\
dol http://dx.doi.org/10.32598/irj.19.3.1406.3
\end{tabular}

Article info:

Received: 04 Jun 2021

Accepted: 16 Aug 2021

Available Online: 01 Sep 2021

Keywords:

Balance, Dynamic neuromuscular stability, Elderly, Gait, Postural control

\section{A B STRACT}

Objectives: Walking and balance control are determining factors in the independence of the elderly because they are the main components of daily physical activity. This study evaluated the effectiveness of Dynamic Neuromuscular Stability (DNS) training on balance and gait function in the elderly.

Methods: This study had a pre-test/post-test design. It was a quasi-experimental study performed on 30 older men aged 60-70 years in Qom Province, Iran, in 2021. The elderly was randomly divided into experimental $(n=15)$ and control $(n=15)$ groups. The Experimental Group (EG) participated in three 45-min sessions of dynamic neuromuscular stability training every week for 6 weeks, and the Control Group (CG) continued their normal daily routine. Berg balance scale (dynamic balance), modified stork stand (static balance), and temporal and spatial gait parameters were used to collect information before and after applying the training protocol. The obtained data were analyzed using ANCOVA statistical method and the paired $t$ test.

Results: There were significant differences between pre-test and post-test in EG regarding the variables of dynamic balance, static balance $(\mathrm{P}=0.001)$, and gait function, but in the $\mathrm{CG}$, no significant difference was observed for these variables $(\mathrm{P}>0.05)$. Also, there was a significant difference between the mean scores obtained in the balance and gait tests between the two groups $(\mathrm{P}<0.05)$, and EG performed better in the post-test.

Discussion: DNS training due to the great variety of movement in different parts of the body and the effect on improving strength, flexibility, range of motion, and physical fitness can also improve balance and gait function in the elderly. According to the present study results, one of the best ways to reduce the costs and physical, psychological, and social problems caused by aging is to use DNS training for the elderly.

\section{* Corresponding Author:}

Mohammad Hani Mansori, MSc.

Address: Department of Physical Education and Sport Sciences, University of Tehran, Kargar Street, Tehran, Iran.

Tel: +98 (935) 5301573

E-mail: haninio74@gmail.com 


\section{Highlights}

- Dynamic neuromuscular stability training improved balance function (static balance, dynamic balance) in the elderly

- Dynamic neuromuscular stability training improved gait function (step length, cadence, width of step, and walking speed) in the elderly.

- Perhaps one of the best ways to reduce the costs and physical, psychological, and social problems associated with aging is to use dynamic neuromuscular stability exercises for the elderly.

\section{Plain Language Summary}

According to the World Health Organization, over the next 30 years, the elderly will make up about $20 \%$ of the world's population, so that by 2050 , the elderly population will reach two billion. With increasing age, changes occur in different body systems, and reports indicate that the aging period is associated with defects in balance, muscle weakness, and decreased reaction speed. These factors cause people to lose their independence in performing one or more daily activities and increase the possibility of injury, resulting in high costs. At the same time, preventive measures and exercise can essentially delay the changes caused by aging. Using dynamic neuromuscular stability exercises due to their effect on physical fitness, strength, range of motion, etc., can help improve balance and gait in the elderly.

\section{Introduction}

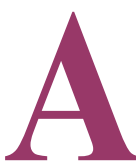

ccording to the definition of the World Health Organization, crossing the age of 60 is known as the aging period [1] Because of the importance of quality of life and improving life expectancy among people, $1.7 \%$ is added to the world's population every year, and this ascending trend for people over 65 and older is $2.5 \%$ [2]. Forecasts show that by 2050 , the aging population will reach two billion people $(25 \%$ of the world's population) [3]. In Iran, according to the last census in 2016, out of a population of 79 million people, nearly 7.5 million are elderly [4]. Middle Eastern countries such as Iran and Turkey are among the countries where population growth is increasing rapidly. Given the increase in the prevalence of older people to $8.5 \%$ in 2018, forecasts show that aging in these areas is expanding rapidly [5]. With aging, changes occur in different body systems, and reports indicate that about $10 \%$ of people lose their independence in performing one or more daily activities by entering old age. This decline in function can be due to psychological factors, environmental conditions, lifestyle, neuromuscular system weakness, changes in functional structures, connective tissue, muscle and muscle strength, neuralgia, changes in senses of vision, atrium, hearing, motor sensation because of increase in age [6].

One of the most severe consequences of old age is the risk of falling, and about one-third of the elderly experi- ence falling one or several times a year [7]. Falling occurs due to some deficiencies in physical fitness factors such as imbalance, muscle weakness, and slow reaction, which ultimately leads to consequences such as poor postural control and changes in gait [8]. Many researchers consider gait and balance control determining factors of the independence of older people's lives because the main components of daily physical activity can be divided into two parts: 1) maintaining balance to maintain body position and spatial orientation and 2) interaction between anatomical components for movement [9] Balance disorder occurs in more than $75 \%$ of people 70 years of age and older [10].

Research has shown that in old age, changes occur in the visual, sensory, atrial, and especially nervous systems as physiological systems involved in the balance of the elderly. These changes expose them to severe damage due to imbalance and gait disorder and increase care and treatment costs $[11,12]$. Balance is a complex motor skill that describes the dynamics of the body in preventing falls [13], and dynamic balance is more affected by aging than static balance [14]. However, maintaining balance and mobility is vital for a healthy lifestyle in the elderly, and in addition to making it possible to perform basic daily activities such as getting up from a chair or climbing stairs, it is a basis for adopting an active lifestyle [15]. These age-related functional changes in balance and posture control reduce the ability to perform daily tasks and gait performance in the elderly and reduce their quality of life [16]. 
Another important consequence of aging is a change in gait pattern and speed, leading to an inability to walk properly [17]. Walking as a fundamental skill is an essential part of daily activities. This skill, which is an indicator for determining the degree of independence in daily affairs, is associated with changes in old age [18]. It has been shown that with aging, a person's walking speed is affected by the length and width of the step [17, 19] so that the walking speed during the $40-80$ decade is reduced by $9 \%-11 \%$ and in fast walking speed by $8 \%$ $18 \%$ [20]. In the elderly, step length, walking height, and speed decrease are closely related to decreased balance. Parvazi et al. also reported less range of motion of lower limb joints, shorter stride length, and greater stride width in the elderly than in the youth [21]. Considering the effects of aging on the reduction of motor and balance function, musculoskeletal structures and the issues mentioned earlier, the prevention of reduced activity levels, overcoming the disabilities of the elderly, and the study of effective factors in changing the quality of their independent life are important. One of these methods is the use of exercise training program, which is a cheap, noninvasive, low-risk, very useful, and purposeful tool for the effectiveness of functional disabilities in the elderly [22], confirmed in previous research. These studies have shown that strengthening and activating any of the factors involved in maintaining balance and gait, such as sensory (vestibular, proprioception) and motor (strength and flexibility) systems, can be a good strategy to prevent gait and balance problems in the elderly [23-25].

Moreover, the muscular and the nervous systems play a significant role in controlling movement, gait, and pattern of movement, especially in the elderly who have limited mobility. One of the exercise rehabilitation techniques is Dynamic Neuromuscular Stabilization (DNS) training, which involves the nervous system and strengthening the muscular system [26]. The rehabilitation should provide a comprehensive program involving all components of physical fitness so that in addition to strengthening the weakening factors in the elderly, it improves balance function and gait performance in the elderly. Evaluation of the effects of DNS exercises has been done to improve balance, performance, and gait parameters on different populations [27, 28], showing the positive effect of these exercises on the above factors. DNS exercises are essential for neuromuscular coordination based on a wide range of strength, range of motion, and proprioception function, including a combination of flexibility, core stability, balance, strength training [29].

More attention should be paid to improving the balance and gait parameters in the elderly. Since the loss of bal- ance and changes in gait pattern are the most common problems of the elderly, which can have physical consequences and impose high costs to families following the fall of the elderly, their motor rehabilitation and increasing their physical activity seem necessary. Considering the novelty of the subject, review of the literature and lack of similar research, and innovation of the mentioned exercises, the researchers decided to evaluate the effects of Dynamic Neuromuscular Stability (DNS) exercises protocol on balance and gait performance in the elderly.

\section{Materials and Methods}

This applied research had a quasi-experimental design. The study was conducted on two groups with 6 weeks of DNS training intervention in the Experimental Group (EG) and non-training intervention in the Control Group (CG). It also used a pre-test and post-test design. The statistical population included all older men over 60 years of age living in Qom City, Iran. A total of 70 people volunteered for the study, and 30 older adults over 60 years were selected based on the inclusion criteria and then randomly divided into two groups of control $(n=15)$ and experimental $(\mathrm{n}=15)$.

A total of 40 people who were not eligible were excluded from the study. The inclusion criteria consisted of the male elderly over the age of 60 , independence in daily activities and no use of assistive devices, no history of cardiovascular diseases, respiratory problems, no severe low back pain in the past 6 months, no history of fractures, and severe injury in the lower limb or any visible abnormality problematic for the research implementation, and voluntary participation in the research. The exclusion criteria included the inability to the training, occurrence of pain during the training protocol, lack of cooperation and withdrawal, and absence in two consecutive sessions and three alternating training sessions. All participants entered the study after filling out the consent form. Also, each participant in the study could withdraw at any stage of the research without paying compensation. To collect data, the subjects were asked to be present at the appointed time and place through announcing the subjects and observing the COVID-19 health-related protocols. To prevent coronavirus infection, the subjects were required to wear masks and gloves during exercise. Only three subjects per hour could participate in the study.

\section{Dynamic balance}

The Berg Balance Scale (BBS) is a test consisting of 14 functional activities that older subjects are responsible for performing, and the examiner scores the appropriate 
score according to the instructions for each activity, and the highest overall score on this scale is 56 [30]. The ICC $(95 \% \mathrm{CI})$ values of $0.93(0.87-0.96)$ and 0.95 (0.92-0.97) were reported for inter-rater and intra-rater reliabilities, respectively, which indicated the validity and reliability of the instrument [30].

\section{Static balance}

The stork balance test was used to assess static balance, and subjects were asked to stand on their upper leg and place the toes on the knee of the upper leg while placing their hands on their waists. Then, with the start command, lift the heel of the upper foot and stand on your toes and maintain the balance without moving the foot or separating the hands from the waist. Maintaining this position (seconds) was considered an individual record. Acceptable levels of intra- and inter-rater reliability and high validity have been reported for the modified stork stand test [31].

\section{Gait function}

Temporal and spatial walking parameters, including step length, step width, and walking pace, were measured by pouring powder to a distance of $10 \mathrm{~m}$ on the ground. Test subjects were asked to walk the indicated distance. The step length was measured by measuring the footprint with a ruler that covers the distance between the heel of one limb and the heel of the next limb. To measure the step width, the distance of two points from the center point of each heel was used as a reference, and the step speed was obtained from the number of steps recorded per minute by a stopwatch [32]. To measure the walking speed of the elderly, the subjects were asked to walk a distance of $10 \mathrm{~m}$ with a maximum speed. Each subject performed this test twice, and his best record was calculated. By dividing this number by the desired distance, the person's walking speed was obtained in $\mathrm{m} / \mathrm{s}$ [33].

\section{Exercise protocol}

DNS training protocol was implemented in 45-min sessions, three sessions per week for six weeks, for the experimental group. These exercises have several models and different levels of training [29]. The training protocol of the experimental group included warm-up exercises (5 minutes), DNS exercises with respiratory correction (40 minutes), and cooling exercises (5 minutes). According to the DNS approach, the main movements with different levels include diaphragmatic breathing (6 levels), lying on your back 90-90 (21 levels), lying on your stomach (9 levels), wrong action (20 levels), sitting on your side (11 levels), sit for miles (11 levels), tripods (13 levels), kneel (11 levels), squat and get up (9 levels) [29]. The first week of training was devoted to training and practicing basic DNS movements. Each week, compared to the previous week, some movement complexity was added. To increase the effectiveness of the exercises during the training period, the overload principle of increasing the number of repetitions and seconds and the type of training was used. It was essential to pay attention to the following points: A) breathing unconsciously through the diaphragm, B) when performing the movement, be careful that the position of the knees is not in the position of varus and valgus, $\mathrm{C}$ ) not having flexion or hyperextension in the back. After completing the 6-week protocol with an interval of one week, the post-test was performed, and the final information was recorded. The control group during this period engaged in their daily activities and normal walking.

After collecting information, the data related to the subjects are analyzed in two sections of descriptive and inferential statistics in SPSS version 22. Descriptive statistics, the ANCOVA test, and the paired sample t test were used for data analysis at a 95\% significance level $(\alpha=0.05)$.

\section{Results}

First, the normality of demographic information and pre-test variables were examined. The Shapiro-Wilk test was used to check the normality of data distribution. This test showed that the data distribution in the two groups was normal $(\mathrm{P}>0.05)$. Then, the independent $t$ test was used to evaluate the homogeneity and uniformity of demographic information and pre-test variables in the EG and CG. The results of this section are presented in Table 1, and it was shown that the two groups in terms of demographic information and the variables of static balance, dynamic balance, length of step, cadence, the width of step, and walking speed were not significantly different in the pre-test stage $(\mathrm{P}>0.05)$.

Therefore, to investigate the effect of DNS exercises on static balance, dynamic balance, and gait function variables at the intergroup level of the two groups (control and experimental), the analysis of covariance (ANCOVA) test was used, and the paired sample $t$ test was used at the intragroup level. The ANCOVA test results are presented in Table 2. Findings of this test by considering the sphericity presumption showed that in static balance $(\mathrm{P}=0.001)$, dynamic balance $(\mathrm{P}=0.001)$, length of step $(\mathrm{P}=0.003)$, cadence $(\mathrm{P}=0.003)$, the width of step $(\mathrm{P}=0.007)$ and walking speed $(\mathrm{P}=0.001)$, a significant difference between 
Table 1. Demographic information of samples (Mean $\pm S D$ ) and data obtained from research variables in the pre-test

\begin{tabular}{|c|c|c|c|c|}
\hline Variables & $\begin{array}{c}\text { Groups ( } n=\text { Each group } \\
15 \text { ) }\end{array}$ & Mean $\pm S D$ & $\mathbf{t}$ & $\mathbf{P}$ \\
\hline \multirow{2}{*}{ Age (y) } & Experimental & $64.66 \pm 3.84$ & \multirow{2}{*}{-0.403} & \multirow{2}{*}{0.690} \\
\hline & Control & $65.13 \pm 2.29$ & & \\
\hline \multirow{2}{*}{ Weight (kg) } & Experimental & $68.40 \pm 1.84$ & \multirow{2}{*}{1.371} & \multirow{2}{*}{0.181} \\
\hline & Control & $67.46 \pm 1.88$ & & \\
\hline \multirow{2}{*}{ Height $(\mathrm{cm})$} & Experimental & $165.20 \pm 4.47$ & \multirow{2}{*}{-0.655} & \multirow{2}{*}{0.518} \\
\hline & Control & $166.26 \pm 4.44$ & & \\
\hline \multirow{2}{*}{ Dynamic balance } & Experimental & $35.93 \pm 2.54$ & \multirow{2}{*}{0.582} & \multirow{2}{*}{0.565} \\
\hline & Control & $35.40 \pm 2.47$ & & \\
\hline \multirow{2}{*}{ Static Balance } & Experimental & $8.53 \pm 0.81$ & \multirow{2}{*}{-0.872} & \multirow{2}{*}{0.391} \\
\hline & Control & $8.80 \pm 0.68$ & & \\
\hline \multirow{2}{*}{ Length of step } & Experimental & $0.4 \pm 0.05$ & \multirow{2}{*}{0.140} & \multirow{2}{*}{0.889} \\
\hline & Control & $0.48 \pm 0.18$ & & \\
\hline \multirow{2}{*}{ Cadence } & Experimental & $84 \pm 1.88$ & \multirow{2}{*}{0.084} & \multirow{2}{*}{0.933} \\
\hline & Control & $83.3 \pm 2.40$ & & \\
\hline \multirow{2}{*}{ Width of step } & Experimental & $0.2 \pm 0.42$ & \multirow{2}{*}{0.477} & \multirow{2}{*}{0.637} \\
\hline & & $0.20 \pm 0.41$ & & \\
\hline \multirow{2}{*}{ Walking speed } & Experimental & $1.64 \pm 0.16$ & \multirow{2}{*}{-0.266} & \multirow{2}{*}{0.793} \\
\hline & Control & $1.67 \pm 0.17$ & & \\
\hline
\end{tabular}

Iranian Rehabilitation Journal

the two groups was observed after six weeks of DNS training. It was determined that the EG performed better compared to the $C G$ by assessing the mean scores.

To investigate the differences in pre-test and post-test in the EG and CG groups separately, the paired sample $t$ test was used (Table 3). In the experimental group before and after participating in the DNS training course, the Mean $\pm \mathrm{SD}$ of dynamic balance were $35.9 \pm 2.54$ and $40.20 \pm 1.78$, respectively, and the Mean \pm SD static balance were $8.53 \pm 0.81$ and $13.23 \pm 0.80$, respectively. As you can see in Table 3, this increase in static balance and dynamic balance was statistically significant in the EG $(\mathrm{P}=0.001)$. Also, a significant difference was observed between the pre-test and post-test stages in the variables of step length, cadence, the width of step, and walking speed in the EG $(\mathrm{P}<0.05)$. However, in the $\mathrm{CG}$, no significant change was observed between the means of static balance, dynamic balance, step length, cadence, the width of step, and walking speed variables before and after the DNS training protocol, and it was not statistically significant $(\mathrm{P}>0.05)$.

\section{Discussion}

This study investigated the effect of six weeks of DNS training on balance function factors and gait performance in the elderly. The results showed a significant difference between the control and exercise groups after applying the exercise intervention (DNS) on the balance function factors and gait performance of the elderly. In the experimental group, DNS training improved balance function and gait function in the elderly. Disorders in the proprioception, visual, vestibular systems, flexibility, range of motion, and strength that occur with aging [34-36] can lead to impaired balance and gait. However, the elderly could improve their stability and balance by exercise training $[25,36]$.

The DNS exercises included different levels of movements in the upper and lower limbs, central body area, breathing, and diaphragm, and so on. Perhaps, at the beginning of the DNS exercises, the increase in strength is due to the neuromuscular coordination created in the muscles [37]. Increased balance function in the elder- 
Table 2. The ANCOVA test results for differences between the two groups

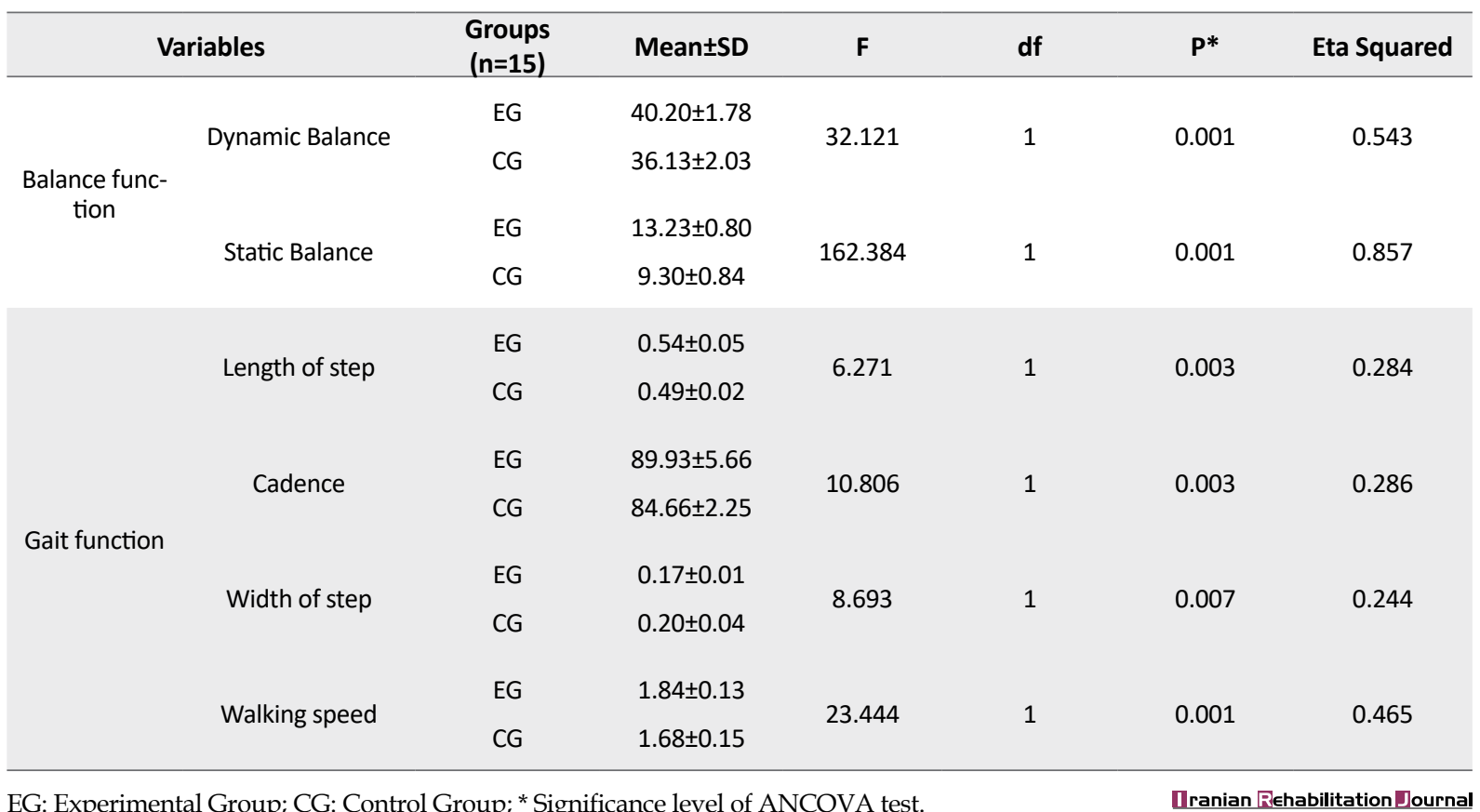

ly after DNS exercises can be due to increased neural mechanisms resulting from exercise, use of more efficient muscle mass, reducing neural inhibitory reactions, and improving and facilitating the transmission of sensory inputs $[37,38]$. These factors can cause structural changes in the muscles that may increase strength. This increase in strength can provide more muscle support for joints such as the thighs and knees and ultimately lead to greater joint stability that improves balance [39].

On the other hand, stabilizing and strengthening the core muscles provides a solid basis for the movement of the limbs. Therefore, strengthening the muscles of the central part of the body, under the influence of the stabilization of the body or the integration system of the spine, can coordinate the organs and the whole body. Proper stability of the spine and central part of the body is provided by the correct breathing pattern [40]. During deep breathing, the diaphragm flattens, and the intra-abdominal pressure increases, which abnormally increases the volume of the abdominal wall and chest with isometric contraction [41].

Also, for a proper gait pattern, the coordination of different muscle groups is required. The intensity and range of motion in flexion, extension, adduction, and abduction to walk even one step depends on muscle strength [42]. Adequate muscle strength facilitates walking, and in contrast, a decrease in muscle strength, especially in old age, leads to a decline in normal functional ability. Therefore, we usually should compensate for this decrease in strength with desirable and effective training programs and thus increase the functional capacity of the elderly [43]. In fact, by improving the strength of the lower and middle limb muscles, the risk of falls in the elderly is reduced, and their ability to walk increases. This type of exercise affects the gait of the elderly by creating neural adaptations. As there is strong coordination between the strength of the quadriceps muscles (extensor) and the hamstring (flexors), knees and especially the dorsiflexors, tibialis anterior (and plantar flexor), and gastrocnemius of the ankle and the ability to control balance in the elderly, it is necessary to design an appropriate exercise program to improve and coordinate the muscles to improve balance and gait in these people.

One of the specific goals of the present study is to improve strength, flexibility, range of motion, and coordination of muscles. Maintaining balance requires the cooperation of different muscle groups. Also, flexion, extension, adduction, and abduction during movement and standing can create accurate movement [44]. Enhancing the strength and flexibility of the quadriceps and thigh extensors in the training protocol of the present study (such as squat, knee and thigh extension and flexion, and the use of Traband) can improve the range of motion the knee and thigh joints. Hence, when standing and walking, compensatory movements are not created by the auxiliary muscle and thus improving balance. Various studies have also shown that limitations in the range of motion of the ankle, knee, and thigh reduce balance [45].

Other possible mechanisms and reasons for the effect of exercises include the balance ability of the elderly 
Table 3. Difference between the mean variables in the pre-test and post-test of both groups

\begin{tabular}{ccccccc}
\hline \multirow{2}{*}{ Variables } & \multicolumn{5}{c}{ Mean \pm SD } \\
\cline { 2 - 7 } & \multicolumn{5}{c}{ Experimental } & \multicolumn{2}{c}{ Control } \\
\cline { 2 - 7 } & Pre-test & Post-test & P* & Pre-test & Post-test & P \\
\hline Dynamic balance & $35.93 \pm 2.54$ & $40.20 \pm 1.78$ & 0.001 & $35.40 \pm 2.47$ & $36.13 \pm 2.03$ & 0.299 \\
\hline Static balance & $8.53 \pm 0.81$ & $13.23 \pm 0.80$ & 0.001 & $8.80 \pm 0.68$ & $9.30 \pm 0.84$ & 0.140 \\
\hline Length of step & $0.49 \pm 0.05$ & $0.54 \pm 0.05$ & 0.011 & $0.48 \pm 0.18$ & $0.49 \pm 0.02$ & 0.235 \\
\hline Cadence & $84 \pm 1.88$ & $89.93 \pm 5.66$ & 0.002 & $83.3 \pm 2.40$ & $84.66 \pm 2.25$ & 0.358 \\
\hline Width of step & $0.21 \pm 0.042$ & $0.17 \pm 0.016$ & 0.013 & $0.20 \pm 0.41$ & $0.20 \pm 0.04$ & 0.789 \\
\hline Walking speed & $1.64 \pm 0.16$ & $1.84 \pm 0.135$ & 0.001 & $1.67 \pm 0.17$ & $1.68 \pm 0.15$ & 0.945 \\
\hline
\end{tabular}

* Significance level of the paired $t$ test.

Mranian Rehabilitation Journa

participants before the exercises. Since the subjects did not regularly participate in physical activities, DNS exercises can increase the physical fitness of these people. So, improving physical fitness in the elderly can be one of the possible causes of improving their balance.

DNS exercises have also been used to improve balance and gait in other populations. Hyun Kim et al. showed that 4 weeks of DNS exercises significantly affect posture control and walking of patients with cerebral palsy [27]. Possible mechanisms of the effect of these exercises are improving the strength of the muscles of the central body and diaphragm in enhancing balance and gait. Zamani et al. (2017) showed that 8 weeks of DNS exercises significantly influenced the balance of patients with multiple sclerosis. The possible mechanisms and reasons for the effect of these exercises include the growth and strengthening of neuromuscular and motor systems [46]. Son et al. (2017) investigated the impact of DNS exercises on balance, function, and gait in people with cerebral palsy and showed that DNS exercises had a significant effect on posture control and walking [28].

Because muscle weakness, inflexibility, and movement control problems are essential factors in poor balance and, consequently, falling, implementing a fitness program is an effective way to prevent falling because exercise and physical activity lead to increased muscle strength, flexibility, and motor control [28]. DNS exercises seem to help improve balance and walk in the elderly due to numerous movements in different parts of the body and the effect on improving strength, flexibility, range of motion, and physical fitness. One of the best ways to reduce the costs and physical, psychological, and social problems is DNS exercises for the elderly.
According to the research findings, it should be noted that improving the balance and gait performance in the elderly, raising the level of physical activity, and motivating participation in training sessions are the strengths of this research. However, the low sample size, the lack of more training groups for comparison, the use of functional tests instead of laboratory tests are some of the weaknesses of this study that should be considered in future research.

One of the limitations of this study is that it did not examine issues related to daily activities, mental status, unavailability of elderly female samples, and comparing them with elderly males. Since the current study was conducted on the elderly, generalizing the results to athletes and people with disabilities should be done with caution. The COVID-19 restrictions also affected the participation of study samples and communication with them. Because of the great importance of balance function factors and walking performance of the elderly and the significant effect of DNS exercises on the elderly, it is recommended to evaluate these exercises on a larger population of older men and women and compare the findings. In this study, functional tests were used to measure gait balance and interaction, and the use of advanced devices such as the Biodex balance system provides more reliable results. Also, the evaluation of range of motion, proprioception, and muscle strength by advanced tools can help in improving the research findings that are recommended to future researchers. The DNS training protocol can also be compared to other training protocols in the elderly.

\section{Conclusion}

Findings from this study show a significant improvement in the effect of DNS exercises on balance function 
factors and gait performance in the elderly. According to research findings, because of the effectiveness of exercises (DNS) on research variables and the importance of balance in reducing the risk of injury to the elderly, it seems that doing these exercises is very useful and increases confidence and improves physical fitness. Perhaps one of the best ways to reduce the physical, psychological, and social costs of aging is to use DNS exercises for the elderly.

\section{Ethical Considerations}

\section{Compliance with ethical guidelines}

The studt was approved by the Ethics Committee of the Faculty of Physical Education and Sports Sciences, University of Tehran (Code: IR.UT.SPORT.REC.1399.042). The subjects were not forced to continue cooperating. At the same time, the principle of confidentiality was observed regarding all information of individuals.

\section{Funding}

The studt was extracted from the $\mathrm{PhD}$ dissertation of the first author at the Department of Pathology and Corrective Movements, Faculty of Physical Education, University of Tehran.

\section{Authors' contributions}

Conceptualization and supervision: Asghar Farhadi and Ibrahim Hassanpour; Methodology: Mohammad hani Mansori; Yousef Moghadas Tabrizi; Investigation, writing - original draft, writing - review \& editing: All authors; Data collection: Mohammad Hani Mansori and Kamal Mohammadkhani; Data analysis: Mohammad hani Mansori; Funding acquisition and Resources: All authors.

\section{Conflict of interest}

The authors declared no conflict of interest.

\section{Acknowledgments}

We appreciate the older participants, the friends who helped us during the tests, and the trainers who helped us with our exercises.

\section{References}

[1] Beissner KL, Collins JE, Holmes H. Muscle force and range of motion as predictors of function in older adults. Physical Therapy. 2000; 80(6):556-63. [DOI:10.1093/ptj/80.6.556] [PMID]
[2] Cohen JE. Human population: The next half century. Science. 2003; 302(5648):1172-5. [DOI:10.1126/science.1088665] [PMID]

[3] Teymoori F, Dadkhah A, Shirazikhah M. Social welfare and health (mental, social, physical) status of aged people in Iran. Middle East Journal of Age and Ageing. 2006; 3(1):39-45. https://www.researchgate.net/profile/Asghar-Dadkhah/ publication/255172751_pdf

[4] Mehri N, Messkoub M, Kunkel S. Trends, determinants and the implications of population aging in Iran. Ageing International. 2020; 45(4):327-43. [DOI:10.1007/s12126-020-09364-z]

[5] Bawazir AA, Aslam M, Osman AF. The impact of population aging on economic growth: Panel data evidence from middle east countries. In: Yilmiz B, editor. Handbook of Research on Economic and Social Impacts of Population Aging. Hershey: IGI Global; 2021. p. 67-86. [DOI:10.4018/978-1-79987327-3.ch005]

[6] Wilson ML, Rome K, Hodgson D, Ball P. Effect of textured foot orthotics on static and dynamic postural stability in middle-aged females. Gait \& Posture. 2008; 27(1):36-42. [DOI:10.1016/j.gaitpost.2006.12.006] [PMID]

[7] Rasteh H, Olyaei G, Abdolvahab M, Jalili M, Jalaei S. [Efficacy of Cawthorne \& Cooksey exercise on balance improvement in elderly persons in Mashhad (Persian)]. Journal of Modern Rehabilitation. 2009; 3(1):49-53. http://mrj.tums. ac.ir/article-1-124-en.html

[8] Shin SS, An DH. The effect of motor dual-task balance training on balance and gait of elderly women. Journal of Physical Therapy Science. 2014; 26(3):359-61. [DOI:10.1589/jpts.26.359] [PMID] [PMCID]

[9] Kimura T, Kobayashi H, Nakayama E, Hanaoka M. Effects of aging on gait patterns in the healthy elderly. Anthropological Science. 2006; 117(1):67-72 [DOI:10.1537/ase.060309]

[10] Dillon CF Gu Q, Hoffman HJ, Ko CW. Vision, hearing, balance, and sensory impairment in Americans aged 70 years and over: United States, 1999-2006. NCHS Data Brief. 2010; (31):1-8. [PMID]

[11] Wiesmeier IK, Dalin D, Maurer C. Elderly use proprioception rather than visual and vestibular cues for postural motor control. Frontiers in Aging Neuroscience. 2015; 7:97. [DOI:10.3389/fnagi.2015.00097] [PMID] [PMCID]

[12] Ballesteros JM, Struijk EA, Rodríguez-Artalejo F, LópezGarcía E. Mediterranean diet and risk of falling in community-dwelling older adults. Clinical Nutrition. 2020; 39(1):27681. [DOI:10.1016/j.clnu.2019.02.004] [PMID]

[13] Shumway-Cook A, Woollacott MH. Motor control: translating research into clinical practice. Philadelphia: Lippincott Williams \& Wilkins; 2007. https://books.google.com/ books?id=BJcL3enz3xMC\&dq

[14] Hanachi P, Kaviani G. [IMPACT of mini trampoline exercise on dynamic balance in elderly women, Tehran, Iran (Persian)]. Hormozgan Medical Journal. 2010; 14(2):148-55 https://www.sid.ir/fa/journal/ViewPaper.aspx?ID=113003

[15] Rose DJ. Fallproof! A comprehensive balance and mobility training program. Champaign: Human Kinetics; 2010. https://books.google.com/books?id=0kLB3yPU8_gC\&dq 
[16] Rezazadeh N, Baluchi R. [The effect of 12 weeks exercise in the water and land on the static and dynamic balance and the risk of falling the old people (Persian)]. Salmand: Iranian Journal of Ageing. 2016; 10(4):140-51. http:// salmandj.uswr. ac.ir/article-1-788-en.html

[17] Kirkwood RN, de Souza Moreira B, Vallone MLDC, Mingoti SA, Dias RC, Sampaio RF. Step length appears to be a strong discriminant gait parameter for elderly females highly concerned about falls: A cross-sectional observational study. Physiotherapy. 2011; 97(2):126-31. [DOI:10.1016/j.physio.2010.08.007] [PMID]

[18] Hosseini SS, Mirzaei B, Panahi M, Rostamkhany H. Effect of aquatic balance training and detraining on neuromuscular performance, balance and walking ability in healthy older men. Middle-East Journal of Scientific Research. 2011 9(5):661-6. https://www.idosi.org/mejsr/mejsr9(5)11/17.pdf

[19] Silsupadol P, Shumway-Cook A, Lugade V, van Donkelaar P, Chou LS, Mayr U, et al. Effects of single-task versus dual-task training on balance performance in older adults: A double-blind, randomized controlled trial. Archives of Physical Medicine and Rehabilitation. 2009; 90(3):381-7. [DOI:10.1016/j.apmr.2008.09.559] [PMID] [PMCID]

[20] Bohannon RW. Comfortable and maximum walking speed of adults aged 20-79 years: Reference values and determinants. Age and Ageing. 1997; 26(1):15-9. [DOI:10.1093/ageing/26.1.15] [PMID]

[21] Proczai R, Bejek Z. Illyés A. Kinamatic and kinetic parameters of healthy elderly people Periodical Polytechnincal Ser Mech Eng J. 2005; 49(1):63-7. https://pp.bme.hu/me/article/view/1342

[22] Maki BE, Sibley KM, Jaglal SB, Bayley M, Brooks D, Fernie GR, et al. Reducing fall risk by improving balance control: Development, evaluation and knowledge-translation of new approaches. Journal of Safety Research. 2011; 42(6):473-85. [DOI:10.1016/j.jsr.2011.02.002] [PMID]

[23] Chehri SM, Yoosefy B, Golchini A. [The effect of eight weeks combined exercises on standing \& walking ability in 65 to 80 years old sedentary males (Persian)]. Nursing And Midwifery Journal. 2015; 13(6):481-9. http://unmf.umsu. ac.ir/article-1-1736-en.html

[24] Dastmanesh S, Sahebzamani M, Karimi MT. [Effect of otago and tai chi exercise programs on balance and risk of falls in elderly men (Persian)]. 2019; 8(1):156-64. [DOI:10.22037/ JRM.2018.111376.1948]

[25] de Oliveira MR, da Silva RA, Dascal JB, Teixeira DC. Effect of different types of exercise on postural balance in elderly women: A randomized controlled trial. Archives of Gerontology and Geriatrics. 2014; 59(3):506-14. [DOI:10.1016/j.archger.2014.08.009] [PMID]

[26] Kolar P. Clinical rehabilitation. Tampa: Alena Kobesová; 2014. https://books.google.com/ books?id=d1vtAgAAQBAJ\&dq

[27] Kim DH, An DH, Yoo WG. Effects of 4 weeks of dynamic neuromuscular stabilization training on balance and gait performance in an adolescent with spastic hemiparetic cerebral palsy. Journal of Physical Therapy Science. 2017; 29(10):18812. [DOI:10.1589/jpts.29.1881] [PMID] [PMCID]

[28] Son MS, Jung DH, You JSH, Yi CH, Jeon HS, Cha YJ. Effects of dynamic neuromuscular stabilization on diaphragm movement, postural control, balance and gait performance in cerebral palsy. NeuroRehabilitation. 2017; 41(4):739-46. [DOI:10.3233/NRE-172155] [PMID]

[29] Frank C, Kobesova A, Kolar P. Dynamic neuromuscular stabilization \& sports rehabilitation. International Journal of Sports Physical Therapy. 2013; 8(1):62-73. [PMID] [PMCID]

[30] Salavati M, Negahban H, Mazaheri M, Soleimanifar M, Hadadi M, Sefiddashti L, et al. The Persian version of the Berg Balance Scale: Inter and intra-rater reliability and construct validity in elderly adults. Disability and Rehabilitation. 2012 34(20):1695-8. [DOI:10.3109/09638288.2012.660604] [PMID]

[31] Sadeghi H, Norouzi H, Karimi Asl A, Montazer M. [Functional training program effect on static and dynamic balance in male able-bodied elderly (Persian)]. Salmand: Iranian Journal of Ageing. 2008; 3(2):565-71. http:/ / salmandj.uswr.ac.ir/ article-1-91-en.html

[32] Cimolin V, Galli M. Summary measures for clinical gait analysis: A literature review. Gait \& Posture. 2014; 39(4):100510. [DOI:10.1016/j.gaitpost.2014.02.001] [PMID]

[33] Lopopolo RB, Greco M, Sullivan D, Craik RL, Mangione KK. Effect of therapeutic exercise on gait speed in communitydwelling elderly people: A meta-analysis. Physical Therapy. 2006; 86(4):520-40. [DOI:10.1093/ptj/86.4.520] [PMID]

[34] Hyun J, Hwangbo K, Lee CW. The effects of pilates mat exercise on the balance ability of elderly females. Journal of Physical Therapy Science. 2014; 26(2):291-3. [DOI:10.1589/ jpts.26.291] [PMID] [PMCID]

[35] Mousavi Sadati SK, Rashidzadeh P. [Effect of cerebellar transcranial direct current stimulation on balance and sensory system of the elderly (Persian)]. Scientific Journal of Rehabilitation Medicine. 2020; 8(4):195-205. [DOI:10.22037/ JRM.2019.111594.2070]

[36] Noruzi Z. [The effect of four weeks of selected balance exercises on reducing the risk of falling inactive elderly women with balance defects (Persian)]. Journal of Motor and Behavioral Sciences. 2020; 3(2):123-7. http://www.jmbs.ir/article_114572.html?lang=en

[37] Yoon HS, Cha YJ, You JSH. The effects of dynamic neuromuscular stabilization on respiratory function, fatigue and activities of daily living in subacute stroke patients: A randomized control trial. NeuroRehabilitation. 2020; 47(4):471-7. [DOI:10.3233/NRE-203231] [PMID]

[38] Francio VT, Boesch R, Tunning M. Treatment of a patient with posterior cortical atrophy (PCA) with chiropractic manipulation and Dynamic Neuromuscular Stabilization (DNS): A case report. The Journal of the Canadian Chiropractic Association. 2015; 59(1):37-45. [PMID] [PMCID]

[39] Mahdieh L, Zolaktaf V, Karimi MT. Effects of Dynamic Neuromuscular Stabilization (DNS) training on functional movements. Human Movement Science. 2020; 70:102568. [DOI:10.1016/j.humov.2019.102568] [PMID]

[40] Cha YJ, Lee JJ, Kim DH, You JSH. The validity and reliability of a dynamic neuromuscular stabilization-heel sliding test for core stability. Technology and Health Care. 2017; 25(5):981-8. [DOI:10.3233/THC-170929] [PMID]

[41] Son MS, Jung D, You JH, Yi CH, Jeon HS, Cha Y. Effects of DNS on diaphragm movement, postural control, balance and gait performance in cerebral palsy. Archives of Physical 
Medicine and Rehabilitation. 2017; 98(10):e62. [DOI:10.1016/j. apmr.2017.08.192]

[42] Devereux K, Robertson D, Briffa NK. Effects of a waterbased program on women 65 years and over: A randomised controlled trial. Australian Journal of Physiotherapy. 2005; 51(2):102-8. [DOI:10.1016/S0004-9514(05)70038-6]

[43] Orr R, de Vos NJ, Singh NA, Ross DA, Stavrinos TM, Fiatarone-Singh MA. Power training improves balance in healthy older adults. The Journals of Gerontology. Series A, Biological Sciences and Medical Sciences. 2006; 61(1):78-85. [DOI:10.1093/gerona/61.1.78] [PMID]

[44] Sadeghi H, Ghasempoor H, Yadegaripoor M, Shojaedin S. [The effect of eight week combined aquatic and non-aquatic training program on the lower extremity strength and gait speed of elderly men (Persian)]. Salmand: Iranian Journal of Ageing. 2013; 7(4):59-66. http://salmandj.uswr.ac.ir/article1-544-en.html

[45] Mohammad Khani K, Karimizadeh Ardekani M, Mansori $\mathrm{MH}$. Investigating the flexibility of the ankle and knee joint and determining its relationship with balance, motor function, and risk of falling in visually impaired individuals Physical Treatments: Specific Physical Therapy Journal. 2021; 11(2):93-102. [DOI:10.32598/ptj.11.2.429.2]

[46] Zamani S, Ganji B, Shahbeigi S. [The effect of eight weeks of DNS training on balance in women with multiple sclerosis (Persian)]. Paper presented at: National Conference on Sports Science Developments in the Field, Health, Prevention and Championship Ministry of Science, Research and Technology. 12 May 2016; Qazvin, Iran. https:/ / civilica.com/doc/556446/ 